\title{
Privacy Guarantees through Distributed Constraint Satisfaction
}

\author{
Boi Faltings, Thomas Léauté and Adrian Petcu \\ boi.faltings, thomas.leaute, adrian.petcu@epfl.ch \\ Artificial Intelligence Laboratory \\ Ecole Polytechnique Fédérale de Lausanne (EPFL) \\ CH-1015 Lausanne, Switzerland \\ http://liawww.epfl.ch/
}

\begin{abstract}
The reason for using distributed constraint satisfaction algorithms is often to allow agents to find a solution while revealing as little as possible about their variables and constraints. So far, most algorithms for DisCSP do not guarantee privacy of this information. This paper describes some simple techniques that can be used with DisCSP algorithms such as DPOP, and provide sensible privacy guarantees based on the distributed solving process without sacrificing its efficiency.
\end{abstract}

\section{Introduction}

Many practical situations require the coordination of actions of different agents. For example, consider allocation of airport takeoff and landing slots. Currently, each airport allocates slots individually [9]. However, airlines need combinations of slots to operate sequences of flights. Similar situations exist when sharing pipelines, electricity grids, and other infrastructure among competing agents.

A consideration that often prohibits such coordinated decisions is the desire to keep coordination constraints private. For example, in airport slot allocation, airlines do not want competitors to find out what routes they intend to fly, as this can be used to counter their strategy. As security breaches are frequent in modern information systems, they would not entrust a central platform with this information either.

One possibility to nevertheless achieve coordination is to use a distributed algorithm where agents are themselves responsible for applying the coordination constraints. Several authors have proposed distributed algorithms for constraint satisfaction and optimization, such as $A B T$ [19], $A W C$ [19], $A A S$ [16], ADOPT [12], OPTAPO [11] and DPOP [13]. Surprisingly, despite the privacy motivation, none of these algorithms gives privacy guarantees, and any particular information could be leaked in the right circumstances.
There is significant earlier work on how to measure the privacy loss of these algorithms. Franzin et al. ([5]) measure privacy loss as the reduction in entropy of other agents' preferences. Maheswaran et al. ([10]) develop a framework called valuations of possible states that measures privacy loss as the degree to which the possible states of other agents are reduced. Greenstadt ([7]) uses this framework to analyze privacy loss of DPOP and ADOPT.

While it is important to measure privacy loss, in practice it is important to be able to give guarantees that certain information is not revealed by an algorithm. Brito ([2, 3]) has developed various methods for reducing privacy loss in search and in particular to protect privacy of a constraint from agents involved in it. Greenstadt ([6]) proposes an algorithm, SSDPOP that uses cryptographic secret sharing to eliminate a major source of privacy loss in DPOP, but does not entirely eliminate privacy loss.

Cryptography provides solutions for secure multiparty computation that can in principle solve constraint satisfaction problems with total privacy. This field focusses in particular on interactions between a small number of agents, such as scheduling a single meeting [8], the millionaire's problem of comparing numbers without revealing them [18], or secure auction protocols [1]. These techniques can in principle be extended to more complex scenarios such as constraint satisfaction, but their complexity quickly becomes unmanageable. Examples using cryptographic circuits are $[15,14]$. Other algorithms perform search and protect values with homomorphic encryption, in particular $[17,20]$. However, such search-based algorithms can leak information through the computation time required to find a solution.

In this paper, we show how an existing algorithm for distributed constraint satisfaction, DPOP, can be adapted to give the required privacy guarantees with no increase in complexity and thus solve problems of realistic size. The techniques can also be applied to search-based algorithms such as ADOPT, with the caveat that additional information 
is leaked through the computation time of the search.

Throughout the paper, we will use the simple example of three agents $A, B$ and $C$, interested in two resources $y$ and $z$. Agent $A$ is interested in getting either one of the two resources (OR constraint), agent $B$ also wants either one, but not both (XOR constraint), and agent $C$ wants either both resources or none (equality constraint). For example, agents could be airlines and resources could be landing slots at different airports. In another interpretation, agents could be oil companies and resources could be storage tanks.

In this example, a solution is for instance to give resource $y$ to agent $A$, resource $z$ to $B$, and no resource to $C$. Note that some information is revealed by the fact that each agent finds out the values its variables take in the final solution: for example $C$ will know that some other agents asked for and obtained either resource $y$ or $z$, since it didn't get its request satisfied. No algorithm can avoid this information loss, so agents that cannot accept it need to avoid it through appropriate modeling or other means.

In a problem with multiple solutions, participants must accept to leak the information associated with any of the solutions, since they cannot control which solution will be reached. We call the information that can be inferred from potential solutions the semi-private information. Importantly, the techniques in this paper do not protect semiprivate information, since agents must accept that this might be leaked by the final solution.

We distinguish four types of privacy guarantees:

Agent privacy: no agent can learn the identity of any other agent unless they share a coordination constraint. For example, agent $A$ cannot discover the identity of agent $B$.

Definition: An algorithm for DisCSP preserves agent privacy when no agent learns the identity of the agent controlling any variable $x_{j}$ that does not share a constraint with another variable $x_{i}$ controlled by this agent.

Topology privacy: no agent can learn anything about topological constructs (constraints, cycles) that do not involve a variable that it has a constraint with. For example, agent $A$ cannot find out that another agent has a constraint over resources $y$ and $z$.

Definition: An algorithm for DisCSP preserves topology privacy if no agent learns about the existence of either constraints or cycles of constraints that do not involve at least one variable it controls.

Constraint privacy: no agent can learn the nature or contents of constraints in which it is not involved in. For example, $A$ cannot find out that $B$ wants $y$ XOR $z$. In an optimization setting, $A$ cannot discover what value $B$ attaches to obtaining $y$ or $z$, in case it has any preference for one resource or the other.

Definition: An algorithm for DisCSP preserves full constraint privacy if an agent does not learn the cost of any particular tuple in a constraint that does not involve any variable it controls, except for semi-private information.

It preserves limited constraint privacy if the information that it can learn about such a tuple is bounded by a threshold $\epsilon$ and $\epsilon$ can be made arbitrarily small by suitable choice of protocol parameters.

Decision privacy: no agent can discover the outcome of any decision that other agents make in the final solution.

Definition An algorithm for DisCSP preserves full decision privacy if no agent can learn the values of any variable that it does not control in the final solution, except for semi-private information.

An algorithm for DisCSP preserves limited decision privacy if no agent can learn the values of any variable that it does not control and is not part of the neighbourhood of a variable it controls, except for semi-private information.

In this paper, we show how the DPOP algorithm can be adapted to provide these four types of privacy guarantees.

\section{Preliminaries}

\subsection{Distributed Constraint Satisfaction}

Definition 1 (DisCSP) $A$ discrete distributed constraint satisfaction problem (DisCSP) is a tuple $\langle\mathcal{A}, \mathcal{X}, \mathcal{D}, \mathcal{C}\rangle$ :

- $\mathcal{A}=\left\{a_{1}, \ldots, a_{k}\right\}$ is a set of agents

- $\mathcal{X}=\left\{x_{1}, \ldots, x_{n}\right\}$ is a set of variables. Each variable $x_{i}$ is controlled by an agent $a\left(x_{i}\right)$

- $\mathcal{D}=\left\{d_{1}, \ldots, d_{n}\right\}$ is a set of finite variable domains

- $\mathcal{C}=\left\{c_{1}, \ldots, c_{m}\right\}$ is a set of constraints, where each constraint $c_{i}$ is a function of scope $\left(x_{i_{1}}, \cdots, x_{i_{l}}\right), c_{i}$ : $d_{i_{1}} \times \ldots \times d_{i_{l}} \rightarrow\{0,1\}$, assigning 0 to feasible tuples, and 1 to infeasible ones. Constraints are known to all agents that control a variable involved in the constraint.

A solution is a complete assignment such that the cost $\sum_{c_{i} \in \mathcal{C}} c_{i}=0$, which is the case exactly when it is consistent with all constraints. We can thus understand these values as a cost. The framework can be extended to partial satisfaction or general constraint optimization by finding an assignment that minimizes the cost and letting constraints be general real functions.

In the following, we assume that all agents know the number $n$ of variables in the problem, that the graph formed by the constraints is connected (otherwise each subproblem can be solved independently), and that all agents that control variables in a given constraint can communicate securely. We also assume that all constraints are either unary or binary, which is a common assumption in the literature that can be made without loss of generality. In a slight abuse of language, we also sometimes indifferently write "variable $x_{i}$ " instead of "agent $a\left(x_{i}\right)$." 
Example: Resource Allocation as DisCSP: To illustrate the methods, we are going to use an example of resource allocation which represents one large class of applications. Other applications include coordination of multiagent plans or distributed interpretation of sensor data, and any other constraint satisfaction problem.

We model resource allocation examples by two sets of variables $x$ and $\hat{x} . x_{a}^{b}$ is controlled by the agent offering resource $b$ and takes value 1 if it allocates resource $b$ to agent $a$ and 0 otherwise. $\hat{x}_{a}^{b}$ is a variable controlled by agent $a$ and constrained to be equal to $x_{a}^{b}$. Three types of constraints exist on these variables:

1. Each resource $b$ can only be assigned to at most one agent, so for any pair of variables $x_{a}^{b}$ and $x_{c}^{b}$, the combination of assignments where they are both assigned 1 is infeasible.

2. Each agent has private constraints on the feasible combinations of resource assignments. For example, since agent $A$ is interested in getting at least one of the resources, then $c_{A}\left(\hat{x}_{A}^{y}=0, \hat{x}_{A}^{z}=0\right)=1$ and $c_{A}=0$ in all other cases.

3. Corresponding $x$ and $\hat{x}$ must have equal values.

Constraints of type 1 and 2 must be kept private to the corresponding agents, for they would otherwise reveal important competitive information to competing agents. As shown in the constraint graph of Figure 1, these can in fact be seen as constraints internal to the agents that define them. The only inter-agent constraints are the constraints of type 3, which should also be kept private to the agents that control the corresponding variables.

\subsection{Depth-First Search (DFS) Trees}

Our algorithm works on a Depth-First Search (DFS) traversal of the constraint graph. It constructs a spanning tree consisting of the constraints used to discover the nodes and linking parents to children by edges called tree edges. All other constraints become back edges that link pseudoparents to pseudochildren. Because of the DFS tree construction, all pseudoparents are also ancestors in the spanning tree.

The separator $S e p_{i}$ of $x_{i}$ is the set of ancestors of $x_{i}$ whose removal disconnects the subtree rooted at $x_{i}$ from the rest of the tree. A node's separator can be determined recursively: for a leaf, it is the union of its parent and all pseudoparents; and for a non-leaf node, the union of its parent, pseudoparents, and its children's separators, minus itself.

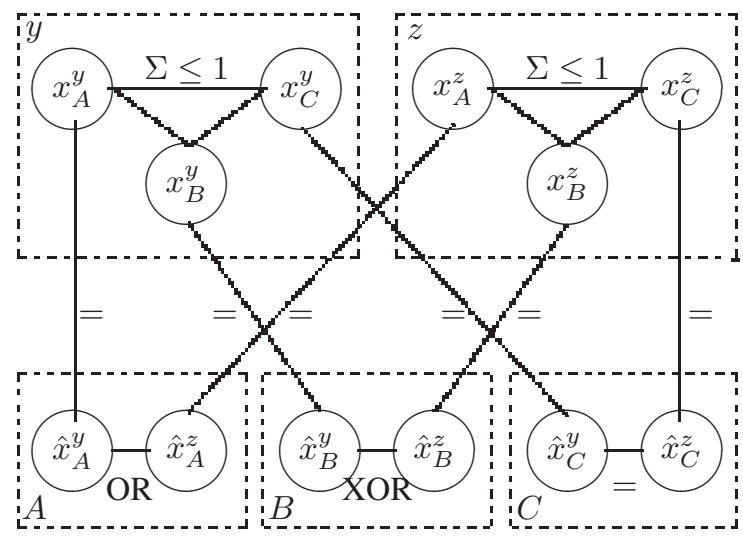

Figure 1. Constraint graph corresponding to the example in which $A$ wants $y$ OR $z$, $B$ wants $y$ XOR $z$, and $C$ wants either both $y$ and $z$ or none.

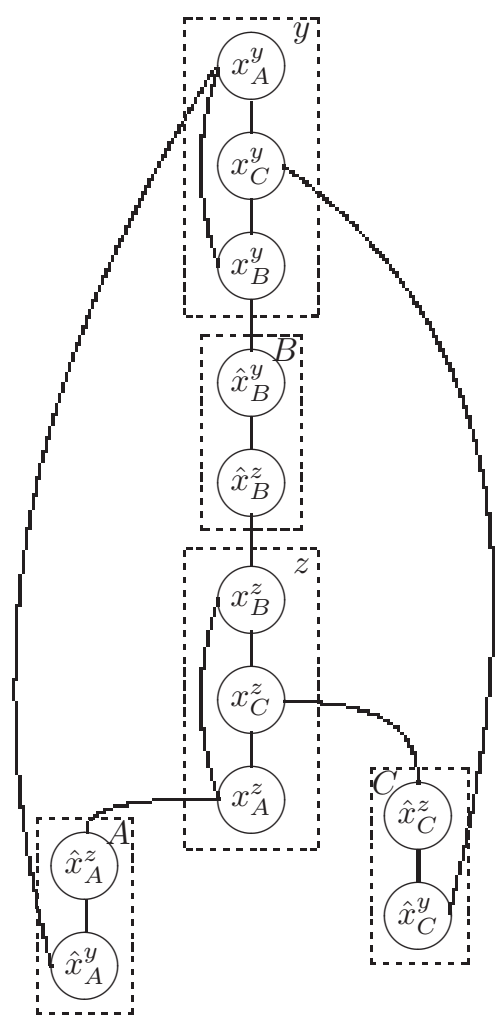

Figure 2. One DFS tree for the example. 


\subsection{DPOP: Distributed Pseudotree Opti- mization Protocol}

DPOP ([13] implements a bucket elimination scheme ([4]) as a distributed protocol. DPOP has 3 phases:

Phase 1 - DFS Tree Generation: A DFS tree is obtained from the constraint graph (as in Figure 2) by first running a decentralized leader election algorithm in order to decide upon the root of the pseudotree. Once the root has been identified, it initiates a decentralized DFS traversal of the graph. As a result, each node consistently labels its neighbors as parent/child or pseudoparent/pseudochild. The DFS tree serves as a communication structure for the other two phases of the algorithm: UTIL messages (Phase 2) travel bottom-up, and VALUE messages (Phase 3) travel top-down, only via tree-edges. Sibling nodes do not exchange any messages.

Phase 2-UTIL Propagation: The agents (starting from the leaves) send UTIL messages to their parents. The subtree of a node $X_{i}$ can influence the rest of the problem only through $X_{i}$ 's separator, $S e p_{i}$. Therefore, a UTIL message contains the optimal cost obtained in the subtree for each instantiation of $S e p_{i}$.

Phase 3 - VALUE Propagation: This is a top-down propagation initiated by the root, when Phase 2 has finished. Each node determines its optimal value based on computation from Phase 2 and the VALUE message it receives from its parent. Then, it sends this value to its children through VALUE messages.

It has been proven in [13] that DPOP produces a linear number of messages. Its complexity lies in the size of the UTIL messages: the largest one is space-exponential in the induced width of the DFS ordering used.

\subsection{Privacy Loss in $D P O P$}

In $D P O P$, privacy is lost along all four dimensions listed in the introduction as a result of executing each of DPOP's three phases. Specifically, in the DFS construction phase, agents learn the identity of all their ancestors (even the ones with whom they are not connected), and the existence and identity of back-edges in the DFS. In the example from Figure 2, agent $A$ learns about agent $B$ as its ancestor, and agent $z$ learns about the existence of agent $y$, and that agent $C$ has a constraint with agent $y$.

Second, in the UTIL propagation phase, all the costs passed in the messages are in clear text, and thus transparent to every agent. Some information is semi-private, for example an agent learn what values are feasible for its own variables under different circumstances. However, the algorithm also leaks consistency information about the variables controlled by other agents: for example, agent $z$ could learn that certain assignments of $x_{A}^{y}$ and $x_{C}^{y}$ are not feasible for $A$ and $C$.

Third, in the VALUE propagation phase, agents learn assignments of other variables. For example, agent $z$ receives the final assignments for variables $x_{A}^{y}$ and $x_{C}^{y}$ in clear text, as this is required for $z$ to make its own final decision.

\section{$3 \quad P-D P O P$ : Privacy Guarantees for DPOP}

This section introduces $P-D P O P$ (Algorithm 1), an algorithm for DisCSP that provides privacy guarantees. $P$ $D P O P$ is derived from $D P O P$ by adding measures that protect privacy during the DFS tree construction, UTIL and VALUE propagation stages. These are described in detail in the following sections.

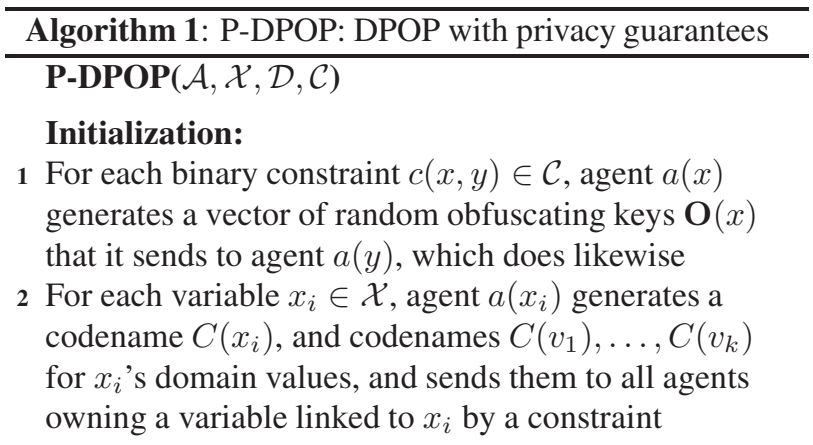

Anonymous DFS construction:

3 Choose root of DFS tree using Algorithm 2

4 Construct DFS labelling using Algorithm 3

\section{UTIL propagation:}

5 Wait for UTIL messages from all children

6 Partially deobfuscate received UTIL messages using known keys and codenames

7 As in $D P O P$, join resulting messages with own unary constraints and binary constraints involving (pseudo-)parents' variables; project $x_{i}$ out

8 Obfuscate result and send to parent

\section{VALUE propagation:}

9 Wait for VALUE message from parent; deobfuscate it 10 Compute optimal value $v_{i}^{*}$ for $x_{i}$

11 Send VALUE messages to all children using the codenames $C\left(x_{i}\right)$ and $C\left(v_{i}^{*}\right)$

\subsection{Privacy in DFS Construction}

The DFS tree is created in a distributed fashion following a message exchange protocol in which agents only communicate with their neighbors in the constraint graph. It consists of two phases: first, a process of anonymous leader election makes one variable as the root of the tree; second, 


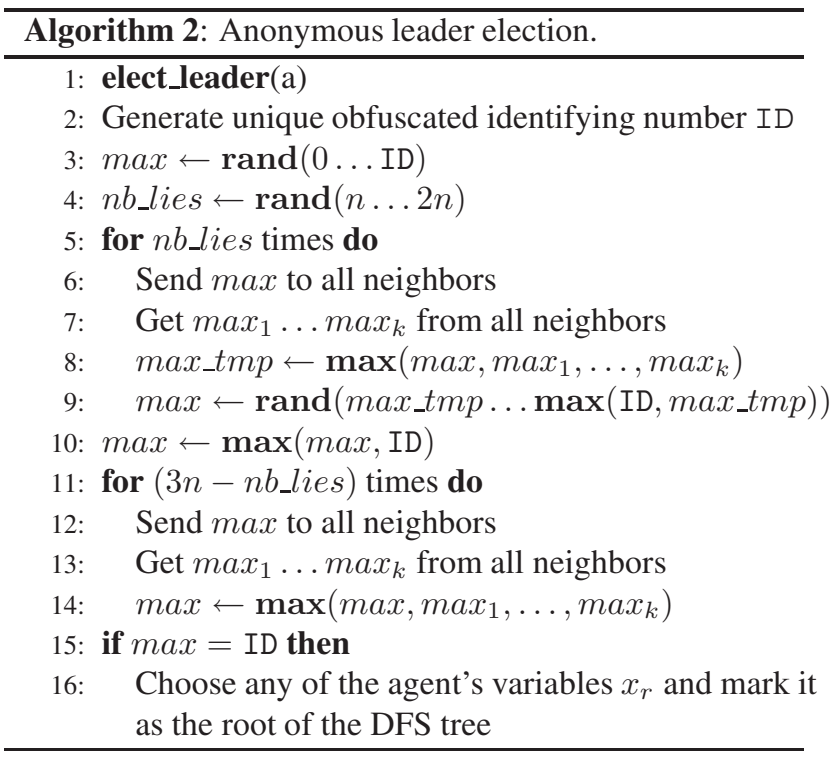

a distributed DFS tree generation protocol categorizes each node's neighbours and thus builds the distributed tree data structure. Both phases guarantee strong agent privacy and topology privacy.

Anonymous leader election (Algorithm 2): To determine the root variable, each agent $a \in \mathcal{A}$ executes the anonymous leader election algorithm given as Algorithm 2 . We assume that each agent has a unique identifier, for example its MAC address. It anonymizes this number, for instance by taking it as exponent in a finite field exponentiation, to generate a unique obfuscated identifying number ID. The protocol then consists, for each agent, in sending its ID number to its neighbors, updating it to the maximum of its ID and its neighbors', and then repeating these two steps such that the knowledge of the maximum ID propagates progressively to all agents. In the end, only one agent has its initial ID number equal to the computed maximum; this agent is the leader, and picks one of its variables $x_{r}$ to be the root of the tree. This protocol converges in $n$ steps, where $n$ is the maximum distance of any two nodes in the graph, for which the number of nodes in the problem is an upper bound. Thus, the algorithm needs to know an upper bound on the number of nodes in the problem, which is reasonable for almost any practical instance.

A shortcoming of this protocol is that the neighbours of the root agent receive the maximum ID in the first cycle, and can thus tell who the root is. This is avoided by first letting the agents give a random lower number and substituting the true ID at an unknown randomly chosen time. In this way, the protocol does not leak any information about the topology of the constraint graph.

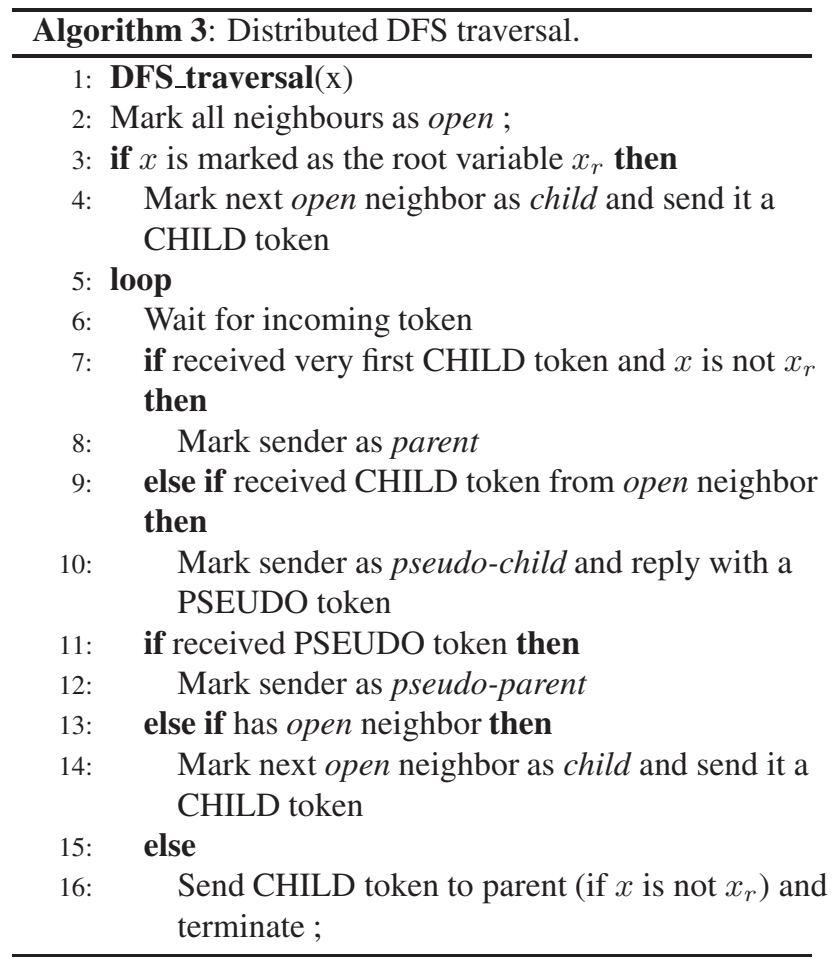

Distributed DFS traversal (Algorithm 3): Once the root variable $x_{r}$ has been marked, the actual DFS construction protocol can be started by executing Algorithm 3 for each node $x \in \mathcal{X}$. Each variable knows its neighbours, which are the variables that it shares a constraint with. It assigns one of the following labels to each neighbor: open, parent, child, pseudo-parent and pseudo-child. Initially, all neighbors are open. The root variable starts by sending a CHILD token that will traverse the constraint graph in DFS order. When a non-root variable receives its very first CHILD token, it marks the sender as its parent, and forwards the token to its next open neighbor, which it marks as a child. When the variable runs out of open neighbors, it resends the CHILD token to its parent, hereby telling it that its whole subtree has been explored, and can proceed with the next phase of the P-DPOP algorithm. When a variable receives the CHILD token from the variable it previously sent it to, it knows it can forward it to its next open neighbor, which it marks as another child. However, if it receives the CHILD token from a different, still open neighbor, it can infer there is a cycle in the constraint graph. It then marks the sender as a pseudo-child, and replies with a special PSEUDO token to notify it of their pseudo-child/pseudo-parent relationship. 
Table 1. Message $U T I L_{A \rightarrow z}$ in $D P O P$ (left) and $P$-DPOP(right).

\begin{tabular}{c|c|c|c|c|c|} 
& $x_{A}^{y}=0$ & $x_{A}^{y}=1$ \\
\hline$x_{A}^{z}=0$ & 1 & 0 \\
\hline$x_{A}^{z}=1$ & 0 & 0 \\
\hline
\end{tabular}$\quad$\begin{tabular}{lll} 
& $\Gamma=\alpha$ & $\Gamma=\beta$ \\
\hline$x_{A}^{z}=0$ & 12346 & 23456 \\
\hline$x_{A}^{z}=1$ & 12345 & 23456 \\
\hline
\end{tabular}

\subsection{Constraint Privacy in UTIL Phase via Obfuscation}

Observe first that if the constraint graph is a tree, then agents only learn about their direct neighbors and so both agent and constraint privacy are guaranteed also during UTIL and VALUE propagation phases.

However, privacy problems can arise from the presence of a back edge. A tree edge in the DFS tree could be called upon to transmit a multidimensional message that refers to another agent elsewhere in the tree (namely, the root of the back edge). For instance, in $D P O P$, agent $A$ would send the message $U T I L_{A \rightarrow z}$ in Table 1(left) to agent $z$, containing references to the agent offering resource $y$, whose identity $A$ might want to hide from $z$, and also and more importantly from possible competing agents that could be asked to relay this information to $y$ (such as agent $B$ ).

We identify the corresponding variable's name as well as its different values by codenames that are only known to the agents at both ends of the back edge. In this way, agents in between do not know what variable the message refers to, nor the possible values for this variable. The codenames are generated by the pseudoparent of the back edge and communicated through a secure channel to its pseudochild at the other end of the back edge. Often, a back edge will link variables belonging to the same agent and the secure channel is trivial to establish. Otherwise, there are numerous secure protocols that can be used for this. In this example, the agent offering resource $y$ decides to represent $x_{A}^{y}=0$ by $\Gamma=\alpha$, and $x_{A}^{y}=1$ by $\Gamma=\beta$, and sends these codenames to agent $A$.

The use of codenames still leaves open the possibility that an agent recognizes the meaning of a message through the values it carries. For example, in a resource allocation problem, an inconsistency usually arises from the nonallocation of a resource. Furthermore, the pattern of consistencies itself can be valuable information that should be protected.

Our solution is to obfuscate constraints by adding large random numbers to their values. If the same number is added uniformly to all values that need to be compared, the results of the comparisons will be unaffected by this obfuscation. This makes it possible to carry out the essential dynamic programming operations on obfuscated numbers
Table 2. Obfuscated UTIL message $U T I L_{C \rightarrow z}$ obtained by using the codenames $\Delta$ for variable $x_{C}^{y}, \gamma$ for 0 and $\delta$ for 1 , and the vector of random numbers $\mathbf{O}\left(x_{C}^{y}\right)=(34567,56789)$.

\begin{tabular}{l|l|l|} 
& $\Delta=\gamma$ & $\Delta=\delta$ \\
\hline$x_{C}^{z}=0$ & 34567 & 56790 \\
\hline$x_{C}^{z}=1$ & 34568 & 56789 \\
\hline
\end{tabular}

Table 3. Join of the two UTIL messages $U T I L_{A \rightarrow z}$ and $U T I L_{C \rightarrow z}$.

\begin{tabular}{cc|c|c|c|c|} 
& \multicolumn{2}{|c|}{$\Delta=\gamma$} & \multicolumn{2}{c|}{$\Delta=\delta$} \\
& $\Gamma=\alpha$ & $\Gamma=\beta$ & $\Gamma=\alpha$ & $\Gamma=\beta$ \\
\hline \multirow{2}{*}{$x_{C}^{z}=0$} & $x_{A}^{z}=0$ & 46913 & 58023 & 69136 & 80246 \\
\cline { 2 - 6 } & $x_{A}^{z}=1$ & 46912 & 58023 & 69135 & 80246 \\
\hline \multirow{2}{*}{$x_{C}^{z}=1$} & $x_{A}^{z}=0$ & 46914 & 58024 & 69135 & 80245 \\
\cline { 2 - 6 } & $x_{A}^{z}=1$ & 46913 & 58024 & 69134 & 80245 \\
\hline
\end{tabular}

while revealing only certain kinds of information.

For instance, when receiving the message $U T I L_{A \rightarrow z}$, the agent offering resource $z$ could infer that there is an OR constraint between its variable $x_{A}^{z}$ and some other variable (represented by the codename $\Gamma$ ). This is a piece of information that agent $A$ might not want to reveal. To obfuscate it, the agent offering resource $y$ sends through a secure channel a vector of secret, large random numbers $\mathbf{O}\left(x_{A}^{y}\right)=(12345,23456)$ to agent $A$, which is going to add them to all costs in its message corresponding to $x_{A}^{y}=0$ and $x_{A}^{y}=1$, respectively. The resulting obfuscated UTIL message is presented in Table 1.

When receiving the obfuscated message $U T I L_{A \rightarrow z}$, the agent is no longer able to compare the two columns, because they have been added two different, unknown numbers. However, it is still able to compare the rows, which is necessary to carry out its local operations on the received cost values. In particular, it can compute the join of the obfuscated message $U T I L_{A \rightarrow z}$ from agent $A$ with the other obfuscated message $U T I L_{C \rightarrow z}$ (Table 2) received from agent $C$, without de-obfuscating them. The result of this operation is presented in Table 3, and is a doubly-obfuscated message with respect to variables $x_{A}^{y}$ and $x_{C}^{y}$.

The agent offering resource $z$ then joins this matrix with its constraint $x_{A}^{z}+x_{B}^{z}+x_{C}^{z} \leq 1$ (which consists in adding 1 to cost values corresponding to $x_{A}^{z}+x_{B}^{z}+x_{C}^{z}>1$ ), and then projects out variables $x_{A}^{z}$ and $x_{C}^{z}$. This projection is done by comparing cost values row-wise, which is feasible without de-obfuscation since the rows have been added the same vector of numbers. The result is presented in Table 4 , and corresponds to the message that is sent to agent $B$. 
Table 4. Utility message $U T I L_{z \rightarrow B}$.

\begin{tabular}{c|c|c|c|c|} 
& \multicolumn{2}{|c|}{$\Delta=\gamma$} & \multicolumn{2}{c|}{$\Delta=\delta$} \\
& $\Gamma=\alpha$ & $\Gamma=\beta$ & $\Gamma=\alpha$ & $\Gamma=\beta$ \\
\hline$\hat{x}_{B}^{z}=0$ & 46912 & 58023 & 69135 & 80245 \\
\hline$\hat{x}_{B}^{z}=1$ & 46913 & 58023 & 69135 & 80246 \\
\hline
\end{tabular}

Table 5. UTIL message $U T I L_{B \rightarrow y}$ *

\begin{tabular}{l|l|l|l|l|} 
& \multicolumn{2}{|c|}{$\Delta=\gamma$} & \multicolumn{2}{c|}{$\Delta=\delta$} \\
& $\Gamma=\alpha$ & $\Gamma=\beta$ & $\Gamma=\alpha$ & $\Gamma=\beta$ \\
\hline$x_{B}^{y}=0$ & 46913 & 58023 & 69135 & 80246 \\
\hline$x_{B}^{y}=1$ & 46912 & 58023 & 69135 & 80245 \\
\hline
\end{tabular}

Upon receipt of this message, agent $B$ is able to infer that the minimum cost achievable by its subtree depends not only on its decisions (i.e. the value assigned to variable $\hat{x}_{B}^{z}$ ), but also on two other variables represented by the codenames $\Gamma$ and $\Delta$. However, it does not know what these codenames refer to. Furthermore, for a given value of $\hat{x}_{B}^{z}$, it does not know how the minimum achievable cost depends on $\Gamma$ and $\Delta$, since it cannot compare columns without knowing the secret numbers that were used to obfuscate them.

As described previously, the agent can still carry out its local computations without de-obfuscating the cost values. In particular, it first joins the received UTIL message with its XOR constraint, which corresponds to adding 1 to cost values corresponding to $\hat{x}_{B}^{y}=\hat{x}_{B}^{z}$. Projecting out variable $\hat{x}_{B}^{z}$ then yields the message $U T I L_{B \rightarrow y}$ (Table 5) that is sent to the agent offering resource $y$. Again, this projection can be carried out without de-obfuscation, since all costs on the same column have been added the same random number, and the projection is done by comparing costs row-wise.

The agent is then able to de-obfuscate the message simply by subtracting the vector of secret random numbers $\mathbf{O}\left(x_{A}^{y}\right)=(12345,23456)$ from the columns corresponding to $(\Gamma=\alpha, \Gamma=\beta)$, and $\mathbf{O}\left(x_{C}^{y}\right)=(34567,56789)$ from $(\Delta=\gamma, \Delta=\delta)$. Finally decoding the codenames yields the cost matrix in Table 6. Joining this with the constraint $x_{A}^{y}+x_{B}^{y}+x_{C}^{y} \leq 1$ (i.e. adding 1 to all costs that violate this constraint) yields the final cost matrix in Table 7.

Based on this cost matrix, the agent can choose one of

Table 6. De-obfuscated UTIL message $U T I L_{B \rightarrow y}$.

\begin{tabular}{c|c|c|c|c|} 
& \multicolumn{2}{|c|}{$x_{C}^{y}=0$} & \multicolumn{2}{c|}{$x_{C}^{y}=1$} \\
& $x_{A}^{y}=0$ & $x_{A}^{y}=1$ & $x_{A}^{y}=0$ & $x_{A}^{y}=1$ \\
\hline$x_{B}^{y}=0$ & 1 & 0 & 1 & 1 \\
\hline$x_{B}^{y}=1$ & 0 & 0 & 1 & 0 \\
\hline
\end{tabular}

Table 7. Join of $U T I L_{B \rightarrow y}$ with the constraint $x_{A}^{y}+x_{B}^{y}+x_{C}^{y} \leq 1$.

\begin{tabular}{c|c|c|c|c|} 
& \multicolumn{2}{|c|}{$x_{C}^{y}=0$} & \multicolumn{2}{c|}{$x_{C}^{y}=1$} \\
& $x_{A}^{y}=0$ & $x_{A}^{y}=1$ & $x_{A}^{y}=0$ & $x_{A}^{y}=1$ \\
\hline$x_{B}^{y}=0$ & 1 & 0 & 1 & 2 \\
\hline$x_{B}^{y}=1$ & 0 & 1 & 2 & 1 \\
\hline
\end{tabular}

the two feasible decisions (with a cost of 0 ), which are to assign its resource $y$ to $A$ or to $B$, respectively.

\subsection{Limited Decision Privacy through the Use of Codenames}

During the VALUE propagation phase, decisions are made and sent down the tree. In order to provide the same level of privacy as during the UTIL propagation phase, variables and values are referred to by their codenames. For instance, if $x_{B}^{y}=0$ is chosen as the optimal decision, then the agent offering resource $y$ sends the message

$$
V A L U E_{y \rightarrow B}=\left\{x_{B}^{y}=0, \Gamma=\beta, \Delta=\gamma\right\}
$$

to agent $B$, which is then not able to learn that resource $y$ was assigned to the competing agent $A$.

In most applications, agents will learn the values chosen for variables they have constraints with anyway, so limited decision privacy is sufficient. For instance, in our example, agent $B$ would eventually be able to infer the value of variable $x_{B}^{y}$ from the value of its variable $\hat{x}_{B}^{y}$ anyway, since they are constrained to be equal. However, we recognize that there may be applications that require full decision privacy.

\subsection{Privacy Properties}

Proposition 1 Algorithms 1,2 and 3 preserve agent privacy.

Proof: All variables are identified by codenames. Codenames of variables are only communicated between agents that control variables in the same constraint, and agents have no other way of learning codenames.

Proposition 2 Algorithms 1,2 and 3 preserve topology privacy.

Proof: Variables are identified by codenames. Thus, both in the leader election and in the DFS tree generation, an agent does not learn anything other than the codename about agents that it is does not share a constraint with. 
Further information can be inferred from the constructed DFS tree. Here, the presence of a backedge shows the existence of a cycle in the constraint graph. Note that, if the backedge occurs in the propagation from variable $x_{i}$, it indicates a cycle that involves this variable $x_{i}$. Thus, it does not leak information about any topological element that $x_{i}$ is not involved in, and so topology privacy is preserved.

\section{Proposition 3 Algorithm 1 preserves limited constraint} privacy.

Proof: Information about constraints is transmitted during the UTIL propagation phase. Privacy could be violated in two ways: (i) by inference from an obfuscated version of a value (or values correlated to it) to the value itself and (ii) by inference from combinations of values obfuscated with the same key. We prove the protection of the obfuscation in this order.

Let $X \in\left\{0,2^{l}\right\}$ identify the cost of a particular tuple (in this case, it is the number of conflicts entailed by that tuple). An eavesdropping agent $A$ may observe $k$ obfuscated versions of this value or other values that are highly correlated with it; let us call these $Y=Y_{1}, . ., Y_{k}$ and assume that they are all known to represent the same value. The principle is that the value could be guessed by considering that the mean of the distribution of the $Y$ is shifted by $X$. Each value is obfuscated with a different and statistically indepedent $Z_{i} \in\left\{0,2^{m}\right\}$. Then the information that $Y_{1}, \ldots, Y_{k}$ gives about $X$ is:

$$
\begin{aligned}
I(Y ; X)=I(X ; Y) & =H(Y)-H(Y \mid X) \\
& =H(Y)-\sum_{i=1}^{k} H\left(Y_{i} \mid X\right) \\
& =H(Y)-\sum_{i=1}^{k} Z_{i}
\end{aligned}
$$

Now $Y_{i} \in\left\{X, X+2^{m}\right\}$ and $H\left(Y_{i}\right) \leq \log \left(X+2^{m}\right)$, and also $H(Y) \leq k \log \left(X+2^{m}\right)$ because of independence. So we have that

$$
\begin{aligned}
I(Y ; X) & \leq k\left(\log \left(X+2^{m}\right)-\log \left(2^{m}\right)\right) \\
& \leq k\left(\log \left(\left(2^{l}+2^{m}\right) / 2^{m}\right)=k \log \left(1+2^{(l-m)}\right)\right.
\end{aligned}
$$

Thus, the bound on the information that the obfuscated observations give about the cost can be made arbitrarily small by increasing $\mathrm{m}$, and we can reach any desired level of privacy.

The second way to infer information from the obfuscated numbers is to exploit the fact that the same random number $Y_{i}$ must be used to obfuscate an entire column of the UTIL message. In particular, the agent that owns a variable $x_{j}$ can tell the differences in cost of different values for this variable. To use this information for inference about particular tuples would require background information about at least one of the values, for example knowing the minimum or maximum cost value. However, this information is not available to the agent; even the distribution of cost values cannot be used to make inferences about minima or maxima as it is self-similar and so does not reveal anything about the absolute values.

The exception is that in the VALUE propagation phase, the agent finds out that a certain combination of value assignments for other variables leads to a consistent solution, thus allowing it to infer that the minimum value in the corresponding column is zero. Consequently, for any other value in the column it can tell that it has $k$ conflicts. For $k=0$ (no conflict), this is semi-private information, as each of them corresponds to a consistent solution of the CSP and would thus be leaked by that solution. When $k \neq 0$, there are special cases such as functional constraints where knowing that a certain value has $k$ conflicts can be used to infer that a value of another connected variable has $k$ or $k-1$ conflicts. However, because of topology privacy, the agent does not know what constraints these conflicts result from, and thus cannot infer anything about their tuples. Hence, the only information that can be gained is semi-private information.

Proposition 4 Algorithm 1 preserves limited decision privacy.

Proof: During VALUE propagation, agents learn decisions for variables that they are either share a constraint with or that are part of a backedge that is part of the separator they control. When they share a constraint with the variable, limited decision privacy is not violated. Variables involved in backedges are identified by codenames, as are the values that they take. Unless they share a constraint, the agent does not know the meaning of either codename, and thus cannot infer either the variable nor the value that it has taken.

Because of constraint privacy, the decision on one variable does not allow inferring the decision of another variable. Thus, limited decision privacy is preserved.

\section{Conclusions}

Distributed constraint satisfaction and optimization has been studied for many years, and privacy has often been cited as a primary reason for using distributed algorithms. In this paper, we show how an existing algorithm for distributed constraint satisfaction, DPOP [13], can be adapted to give the required privacy guarantees with no increase in algorithmic complexity. Note however that certain heuristics, for example for generating good DFS orderings, interfere with privacy and this could hurt efficiency. However, 
compared with earlier cryptographic solutions the algorithm is still much more efficient and can thus solve problems of realistic size.

While the method we presented does not guarantee complete privacy, we observe that the level of privacy afforded by this algorithm is sufficient for almost all application scenarios. For example, in slot allocation airports will want to know what airlines are asking for what slots, and thus the constraints are known to all involved agents anyway. Likewise, in the solution it will become obvious which airline was allocated which slot by observing their actual operations, so there is no need to protect the privacy of values. On the other hand, it is important that competing airlines do not learn about the constraints of others, and this is protected by the algorithm we proposed. A similar situation exists in combinatorial auctions and other resource allocation problems. For coordination problems, it is clear that agents must know their coordination constraints and the decisions taken on coordination with other agents.

We note that the methods described here work equally well for partial constraint satisfaction or even general optimization when the scope of constraint valuations is extended from $\{0,1\}$ to the real numbers. However, such settings pose additional issues of self-interest as agents will have an interest to drive solutions towards those that satisfy their interest best. These can be addressed with economic mechanisms but these are beyond the scope of this paper.

The methods described here can be also be applied in the context of other distributed constraint satisfaction algorithms. For example, obfuscation and codenames could be used with search algorithms such as ADOPT and NCBB. However, as pointed out in [15], such algorithms leak information through the runtime of the solving process itself, so the value of privacy guarantees is not as clear.

\section{References}

[1] F. Brandt. Fully private auctions in a constant number of rounds. In Financial Cryptography, pages 223-238, 2003.

[2] I. Brito and P. Meseguer. Distributed forward checking. In Proceedings of the Nineth International Conference on Principles and Practices of Constraint Programming (CP'03), volume 2833, pages 801-806, Kinsale, Ireland, September 29-October 3 2003. Springer Berlin / Heidelberg.

[3] I. Brito and P. Meseguer. Distributed forward checking may lie for privacy. In Proceedings of the Nineth International Workshop on Distributed Constraint Reasoning (CPDCR'07), Providence, RI, USA, September 232007.

[4] R. Dechter. Constraint Processing. Morgan Kaufmann, 2003.

[5] M. S. Franzin, E. C. Freuder, F. Rossi, and R. Wallace. Multi-agent constraint systems with preferences: Efficiency, solution quality, and privacy loss. Computational Intelligence, 20(2):264-286, May 2004.
[6] R. Greenstadt, B. Grosz, and M. D. Smith. SSDPOP: Using secret sharing to improve the privacy of DCOP. In Proceedings of the Nineth International Workshop on Distributed Constraint Reasoning (CP-DCR'07), Providence, RI, USA, September 232007

[7] R. Greenstadt, J. P. Pearce, and M. Tambe. Analysis of privacy loss in distributed constraint optimization. In Proceedings of the Twenty-First National Conference on Artificial Intelligence (AAAI'06), pages 647-653, Boston, Massachusetts, U.S.A., July 16-20 2006. AAAI Press.

[8] T. Herlea, J. Claessens, B. Preneel, G. Neven, F. Piessens, and B. D. Decker. On securely scheduling a meeting. In Sec '01: Proceedings of the 16th international conference on Information security: Trusted information, pages 183-198, 2001.

[9] International Air Transport Association (IATA). Worldwide Scheduling Guidelines, 12th edition, December 2005.

[10] R. T. Maheswaran, J. P. Pearce, E. Bowring, P. Varakantham, and M. Tambe. Privacy loss in distributed constraint reasoning: A quantitative framework for analysis and its applications. Autonomous Agents and Multi-Agent Systems (JAAMAS), 13(1):27-60, July 2006.

[11] R. Mailler and V. Lesser. Solving distributed constraint optimization problems using cooperative mediation. Proceedings of Third International Joint Conference on Autonomous Agents and MultiAgent Systems (AAMAS 2004), 1:438-445, 2004.

[12] P. J. Modi, W.-M. Shen, M. Tambe, and M. Yokoo. ADOPT: Asynchronous distributed constraint optimization with quality guarantees. AI Journal, 161:149-180, 2005.

[13] A. Petcu and B. Faltings. DPOP: A scalable method for multiagent constraint optimization. In Proceedings of the 19th International Joint Conference on Artificial Intelligence, IJCAI-05, pages 266-271, Edinburgh, Scotland, Aug 2005.

[14] M.-C. Silaghi, B. Faltings, and A. Petcu. Secure combinatorial optimization simulating DFS tree-based variable elimination. In 9th Symposium on Artificial Intelligence and Mathematics, Ft. Lauderdale, Florida, USA, Jan 2006.

[15] M. C. Silaghi and D. Mitra. Distributed constraint satisfaction and optimization with privacy enforcement. In Proceedings of the 2004 IEEE/WIC/ACM International Conference on Intelligent Agent Technology (IAT'04), pages 531-535, Beijing, China, 2004. IEEE Computer Society.

[16] M.-C. Silaghi, D. Sam-Haroud, and B. Faltings. Asynchronous search with aggregations. In AAAI/IAAI, pages 917-922, Austin, Texas, 2000.

[17] K. Suzuki and M. Yokoo. Secure generalized vickrey auction using homomorphic encryption. Financial Cryptography, 2742:239-249, 2003.

[18] A. C.-C. Yao. Protocols for secure computations. In FOCS, pages 160-164, 1982.

[19] M. Yokoo and K. Hirayama. Algorithms for distributed constraint satisfaction: A review. Autonomous Agents and Multi-Agent Systems, 3(2):185-207, 2000.

[20] M. Yokoo, K. Suzuki, and K. Hirayama. Secure distributed constraint satisfaction: reaching agreement without revealing private information. Artif. Intell., 161(1-2):229-245, 2005 . 